\title{
Plasma Adipokine Levels and Their Association with Overall Burden of Painful Joints among Individuals with Hip and Knee Osteoarthritis
}

\author{
Anthony V. Perruccio, Nizar N. Mahomed, Vinod Chandran, and Rajiv Gandhi
}

\begin{abstract}
Objective. To investigate the association between plasma adipokine levels and the burden of painful joints among individuals with hip and knee osteoarthritis (OA).

Methods. Adipokines (leptin, adiponectin, adipsin, resistin) were determined by ELISA ( $\mathrm{n}=78)$. Individuals reported painful joints on a homunculus. Associations were examined by sex-stratified Poisson analyses.

Results. Adjusted for age, body mass index, and hip/knee OA, higher leptin and adiponectin and lower adipsin levels were associated with greater painful joint burden (i.e., counts) among women $(\mathrm{p}<0.01)$. Among men, higher resistin levels were associated with lower counts $(\mathrm{p}=0.03)$.

Conclusion. Findings support the likelihood of a systemic-dependent sex-specific pain burden among individuals with OA. (First Release Dec 15 2013; J Rheumatol 2014;41:334-7; doi:10.3899/jrheum.130709)
\end{abstract}

Key Indexing Terms: OSTEOARTHRITIS

\section{ADIPOKINES}

While the etiology of osteoarthritis (OA) is not well understood $^{1}$, obesity has long been recognized as an important risk factor. Although the underlying mechanisms in this case are not fully known, mechanical factors are viewed as a likely important link for weight-bearing joints ${ }^{2}$. Findings of positive associations between obesity and $\mathrm{OA}$ in non-weight-bearing joints have suggested systemic links as well ${ }^{1,3,4}$. Finally, the common presence of multiple symptomatic joints (weight-bearing and otherwise) among individuals with late-stage $\mathrm{OA}$ in at least 1 joint ${ }^{5,6}$ further contributes to a broader view that there are systemic components to $\mathrm{OA}^{7}$.

White adipose tissue has been recognized as an important endocrine organ that secretes a wide variety of biologically active adipokines ${ }^{8,9}$. Thus, the metabolic link between

From the Arthritis Program, Toronto Western Hospital, University Health Network; Institute of Health Policy, Management and Evaluation; Toronto Musculoskeletal Centre, University of Toronto; Division of Orthopedic Surgery, Department of Surgery, and Division of Rheumatology, Department of Medicine, University of Toronto, Toronto, Ontario, Canada.

A.V. Perruccio, PhD, Arthritis Program, Toronto Western Hospital, University Health Network, and Institute of Health Policy, Management and Evaluation, and Toronto Musculoskeletal Centre, University of Toronto; N.N. Mahomed, MD, DSc, Arthritis Program, Toronto Western Hospital, University Health Network, and Division of Orthopedic Surgery, Department of Surgery, University of Toronto; V. Chandran, $M D, P h D$, Arthritis Program, Toronto Western Hospital, University Health Network, and Division of Rheumatology, Department of Medicine, University of Toronto; R. Gandhi, MD, MSc, Arthritis Program, Toronto Western Hospital, University Health Network, and Division of Orthopedic Surgery, Department of Surgery, University of Toronto.

Address correspondence to Dr. A.V. Perruccio, Arthritis Program, Toronto Western Hospital, 399 Bathurst St., East Wing, 1st Floor, Room 449, Toronto, ON M5T 2S8, Canada.E-mail: perrucci@uhnres.utoronto.ca Accepted for publication October 17, 2013.
HIP KNEE JOINTS

obesity and OA has focused predominantly on adipokines. However, the focus has nearly exclusively been on associations with single joints. The association between joint pain, the primary symptom of $\mathrm{OA}$, and adipokines also has been examined to some extent ${ }^{10,11,12}$. Again, however, the focus has been on individual joints; the extent of overall symptomatic joint burden has not been considered. We investigated the association between plasma levels of adipokines and the extent of painful joint involvement among patients with endstage hip and knee OA. Because differential associations between obesity and OA have been reported between men and women ${ }^{13,14}$, our investigation was carried out separately in men and women.

\section{MATERIALS AND METHODS}

Subjects. Seventy-eight patients with late-stage hip or knee OA scheduled for joint replacement surgery were consecutively recruited from an academic hospital in Toronto, Canada. Eligibility criteria included being $\geq$ 18 years of age and having the ability to read and comprehend English. Individuals with inflammatory arthritis, those being treated for inflammatory conditions, or those with posttraumatic arthritis were ineligible. Participants were identified and diagnosis confirmed radiographically by participating surgeons.

The study was approved by the University Health Network Research Ethics Board. Written informed consent was obtained from all patients. A study health survey was completed prior to surgery.

Study outcome. Patients were asked to indicate on a homunculus all joints that were painful on most days for at least a month in the past 12 months. A count score was developed of symptomatic regions (e.g., no distinction between 1 and 2 hips; neck, spine, shoulders, elbows, wrists, hands, hips, knees, ankles, feet) not including the surgical joint (possible range of regional symptomatic joint count, 0-9).

The questionnaire also included height and weight, used to calculate body mass index (BMI; $\mathrm{kg} / \mathrm{m}^{2}$ ), and demographic characteristics including age and sex. An indicator variable reflecting presence of knee/hip OA was retained.

Personal non-commercial use only. The Journal of Rheumatology Copyright $@$ $\subsetneq 2014$. All rights reserved 
Laboratory methods. Fasting blood was obtained from all patients prior to surgery, stored at $-80^{\circ} \mathrm{C}$, and analyzed in batch. Plasma concentrations of adipokines were quantitated by ELISA kits according to manufacturers' instructions (human leptin multiplex ELISA Adipokine Panel 2, Bio-Rad, and adiponectin, resistin, and adipsin multiplex ELISA Adipokine Panel 1, Bio-Rad). Samples were diluted as appropriate and assayed in duplicate in the same run

Analysis. Analyses were sex-stratified. Mean age and median values of BMI and adipokines are presented for the sample. Bivariable associations between BMI and adipokine levels were examined using Spearman's correlation coefficient. Poisson regression analysis for count data (i.e., regional painful joint count) was used to assess the multivariable adjusted (age, BMI, knee/hip, adipokines) association between each adipokine concentration level and regional joint count. For comparative interpretability of regression estimates, the effects for leptin, adiponectin, and resistin represent effects for every 5-unit increase in concentration, while for adipsin it is for every unit increase. This was based on the average range of each adipokine concentration distribution, such that the estimates on average represent the effect of a decile increase over the respective concentration range.

\section{RESULTS}

Forty-five women and 33 men were enrolled. Sample descriptions are presented in Table 1. Regional symptomatic joint count (not including surgical joint) ranged from 0 to 8 among women, and from 0 to 5 among men. For adiponectin, resistin, adipsin, and leptin, the intraassay coefficients of variation were $<11.7 \%,<10.1 \%,<5.2 \%$, and $<5.4 \%$, respectively.

Significant Spearman's correlations were found between BMI and adipokine levels. Among women: leptin $(\rho=0.58$, $\mathrm{p}<0.001)$, adiponectin $(\rho=-0.15, \mathrm{p}=0.34)$, adipsin $(\rho=$ $0.38, \mathrm{p}=0.011)$, and resistin $(\rho=0.37, \mathrm{p}=0.013)$; and among men: leptin $(\rho=0.68, p<0.001)$, adiponectin $(\rho=$ $0.06, p=0.73)$, adipsin $(\rho=0.14, p=0.44)$, and resistin $(\rho=0.31, p=0.045)$.

Results from adjusted analyses are presented in Table 2. The fit of the models was tested by way of goodness-of-fit chi-square test. For both women and men, the Poisson model form fit the data reasonably well $(\mathrm{p}=0.0604$ and 0.1462 , respectively). Sex-stratified analyses were justified on the basis of finding significant interactions (data not

Table 1. Sample description.

\begin{tabular}{|c|c|c|}
\hline & $\begin{array}{l}\text { Female, } \\
\mathrm{n}=45\end{array}$ & $\begin{array}{l}\text { Male, } \\
\mathrm{n}=33\end{array}$ \\
\hline \multirow[t]{2}{*}{$\begin{array}{l}\text { Age, yrs, mean }( \pm S D) \text {, } \\
\quad \text { range }\end{array}$} & $\begin{array}{c}64.7(10.1) \\
43.9-82.8\end{array}$ & $\begin{array}{c}61.4(11.3) \\
43.7-88\end{array}$ \\
\hline & Median (range) & Median (range) \\
\hline BMI & $32.5(21.1-52.4)$ & $28.7(20.2-48.5)$ \\
\hline Leptin, ng/ml & $26.3(4.4-66.7)$ & $7.1(2-38.9)$ \\
\hline Adiponectin, $\mu \mathrm{g} / \mathrm{ml}$ & $18(4.5-54.7)$ & $12.4(2.5-47.6)$ \\
\hline Adipsin, $\mu \mathrm{g} / \mathrm{ml}$ & $3.9(2.6-15.2)$ & $3.5(0.8-6.5)$ \\
\hline Resistin, ng/ml & $26.6(14.4-47.4)$ & $26.1(8.7-52)$ \\
\hline
\end{tabular}

BMI: body mass index. shown) between sex and each adipokine level (all $\mathrm{p}<0.04$ ) except adipsin. Overall model fit was poor when interactions were not considered (goodness-of-fit test: chi-square $=113.4454$, df $=67, \mathrm{p}=0.0003$ ).

Among women, adjusted for age, BMI, and knee/hip OA, each $5 \mathrm{ng} / \mathrm{ml}$ increase in leptin and $5 \mu \mathrm{g} / \mathrm{ml}$ increase in adiponectin was significantly associated with a $15 \%$ and $18 \%$ increase, respectively, in regional symptomatic joint count. On the other hand, for each $\mu \mathrm{g} / \mathrm{ml}$ increase in adipsin, regional symptomatic joint count was significantly reduced by $15 \%$. Among men, only resistin was significantly associated with regional symptomatic joint count in adjusted analyses. Each $5 \mathrm{ng} / \mathrm{ml}$ increase in resistin was associated with a $15 \%$ decrease in regional symptomatic joint count.

\section{DISCUSSION}

Findings from our present study suggest that among individuals with hip and knee OA, there may be a "dose-response" association between overall painful joint burden and plasma levels of adipokines. However, differences in association were found between women and men.

Adipokines are emerging as modulators of rheumatic diseases by promoting and perpetuating inflammatory responses. While it is unknown whether the joint pain reported by our study participants is OA-related, other than for their hip or knee, leptin levels have been shown to be associated with pain severity among individuals with hand $\mathrm{OA}^{15}$. As well, leptin has been shown to act as a proinflammatory agent ${ }^{16}$ with catabolic effects in joints affected by $\mathrm{OA}^{17}$. In recent work, serum leptin has been shown to be associated with prevalent and incident knee OA among women $^{18}$, and found to be positively correlated with the severity of knee $\mathrm{OA}^{19}$. In cross-sectional analyses, Ding, et al reported a negative association between serum leptin levels and knee cartilage volume, and Stannus, et al reported a positive association between serum leptin and hip joint space narrowing ${ }^{20,21}$. In both studies, the associations were more notable among women. Within the present sample, composed wholly of individuals with severe hip and knee OA, higher plasma leptin level was associated with overall greater painful joint burden among women. Among men, associations trended toward being negative, though not significantly.

The importance of adiponectin in the pathogenesis of OA has been supported by clinical observations. In patients with OA, plasma adiponectin levels have been reported significantly higher as compared to healthy controls ${ }^{22}$, and were also higher among women with erosive hand $\mathrm{OA}$ as compared to those with nonerosive $\mathrm{OA}^{23}$. Nevertheless, the literature is inconsistent in this regard ${ }^{15}$. We report that among women with severe hip and knee OA, plasma levels of adiponectin were associated with overall greater painful joint burden. The differences in findings between studies are likely consequences both of varying study designs and of

Personal non-commercial use only. The Journal of Rheumatology Copyright @ 2014 . All rights reserved. 
Table 2. Multivariable adjusted associations between regional painful joint count (outcome) and adipokines; Poisson analysis.

\begin{tabular}{|c|c|c|c|c|}
\hline Predictors & $\begin{array}{l}\text { Count } \\
\text { Ratio }\end{array}$ & Lower $95 \%$ CL & Upper $95 \%$ CL & $\mathrm{p}$ \\
\hline \multicolumn{5}{|l|}{ Women } \\
\hline Age & 1.03 & 1.01 & 1.06 & 0.020 \\
\hline BMI & 1.01 & 0.97 & 1.05 & 0.541 \\
\hline Knee vs hip & 1.25 & 0.76 & 2.05 & 0.373 \\
\hline Leptin (per 5 ng/ml) & 1.15 & 1.05 & 1.28 & 0.002 \\
\hline Adiponectin (per $5 \mu \mathrm{g} / \mathrm{ml})$ & 1.18 & 1.06 & 1.31 & 0.003 \\
\hline Adipsin (per $1 \mu \mathrm{g} / \mathrm{ml})$ & 0.85 & 0.75 & 0.96 & 0.009 \\
\hline Resistin (per 5 ng/ml) & 1.06 & 0.93 & 1.20 & 0.361 \\
\hline \multicolumn{5}{|l|}{ Men } \\
\hline Age & 1.02 & 0.98 & 1.05 & 0.402 \\
\hline BMI & 1.03 & 0.93 & 1.14 & 0.533 \\
\hline Knee vs hip & 0.92 & 0.43 & 1.95 & 0.818 \\
\hline Leptin (per 5 ng/ml) & 0.86 & 0.67 & 1.11 & 0.249 \\
\hline Adiponectin (per $5 \mu \mathrm{g} / \mathrm{ml}$ ) & 0.87 & 0.72 & 1.05 & 0.156 \\
\hline Adipsin (per $1 \mu \mathrm{g} / \mathrm{ml})$ & 0.96 & 0.79 & 1.18 & 0.719 \\
\hline Resistin (per 5 ng/ml) & 0.85 & 0.74 & 0.99 & 0.031 \\
\hline
\end{tabular}

CL: confidence limits; BMI: body mass index.

variability in disease stages across samples ${ }^{15}$. As well, the association between adiponectin and pain specifically is not well understood.

Serum resistin levels have been shown to be higher among individuals with severe knee $\mathrm{OA}$ as compared to controls with no $\mathrm{OA}^{24}$. In that study, female sex was associated with higher leptin and adiponectin serum levels, but no association was found between sex and resistin levels. Similarly, in our present study, serum levels of leptin and adiponectin were higher in women, although little difference was observed between sexes for resistin and adipsin. We found that serum resistin level was significantly associated with regional symptomatic joint count only in men, with higher levels associated with a lower overall count. While previous work has not examined serum levels as they relate to overall symptomatic joint burden, among women, higher serum resistin levels have been reported to be associated with radiographic changes in hand $\mathrm{OA}^{25}$. Nevertheless, the role (or lack thereof) of resistin in OA pathogenesis and its association with pain is not clear ${ }^{15}$. The differences we identify between sexes may explain some of the inconsistencies in the literature, particularly where samples are comprised and analyzed considering men and women together. Finally, minimal work has examined the role of adipsin in OA. Among patients scheduled to undergo knee replacement, adipsin in the synovial fluid has been characterized as antiinflammatory ${ }^{26}$, and serum levels have been shown to be higher among individuals with OA compared to those without ${ }^{27}$.

These study results further support the view that systemic effects may be operational among individuals with OA. Unlike previous studies focused exclusively on single joints, this work suggests that overall symptomatic joint burden among individuals with established $\mathrm{OA}$ in the hip or knee may reflect an underlying metabolic systemic phenotype, and further suggests that the characteristics of this phenotype may be sex-specific. Interestingly, with adipokine levels considered, BMI did not exhibit an independent effect on symptomatic joint count. We note, however, that height and weight were self-reported. The extent to which individuals overestimate their height and/or underestimate their weight influences BMI estimates downward. Nevertheless, this is unlikely to affect estimates relating adipokine levels and symptomatic joint count.

The number of patients in our study was relatively small, precluding the drawing of definitive conclusions on the association between circulating levels of adipokines and the extent of symptomatic joint burden. Despite the small size, statistically significant associations were observed, and thus further investigations are warranted. While all patients had severe hip or knee OA, duration of disease was not assessed, and thus uncontrolled for in adjusted analyses. These were cross-sectional findings, and thus causality could not be determined; higher or lower adipokine concentrations can be the cause or the result of OA and/or joint pain (number of joints reported on a homunculus as painful does not mean these joint regions are necessarily affected by the disease). Finally, adipokines have isoforms, and different isoforms may have different biological properties. However, in our study we assessed only totals.

Joint pain in OA as a systemic disorder entails a need to reevaluate $\mathrm{OA}$ treatment and management strategies, with consideration for multimodal approaches. Systemic factors may also help explain high levels of comorbidity in OA populations and may be targets for comorbidity prevention. 


\section{ACKNOWLEDGMENT}

The authors gratefully acknowledge Fawnda Pellett and Fatima Abji of Dr. Dafna Gladman's research laboratory at the Toronto Western Hospital for performing the serum assays.

\section{REFERENCES}

1. Felson DT. An update on the pathogenesis and epidemiology of osteoarthritis. Radiol Clin North Am 2004;42:1-9.

2. Felson DT. Weight and osteoarthritis. Am J Clin Nutr 1996;63 Suppl 3:430S-432S.

3. Rai MF, Sandell LJ. Inflammatory mediators: tracing links between obesity and osteoarthritis. Crit Rev Eukaryot Gene Expr 2011;21:131-42.

4. Toussirot E, Streit G, Wendling D. The contribution of adipose tissue and adipokines to inflammation in joint diseases. Curr Med Chem 2007;14:1095-100.

5. Hawker GA, Badley EM, Borkhoff CM, Croxford R, Davis AM, Dunn $\mathrm{S}$, et al. Which patients are most likely to benefit from total joint arthroplasty? Arthritis Rheum 2013;65:1243-52.

6. Perruccio AV, Power JD, Evans HM, Mahomed SR, Gandhi R, Mahomed NN, et al. Multiple joint involvement in total knee replacement for osteoarthritis: effects on patient-reported outcomes. Arthritis Care Res 2012;64:838-46.

7. Berenbaum F. Osteoarthritis as an inflammatory disease (osteoarthritis is not osteoarthrosis!). Osteoarthritis Cartilage 2013;21:16-21.

8. Kershaw EE, Flier JS. Adipose tissue as an endocrine organ. J Clin Endocrinol Metab 2004;89:2548-56.

9. Trujillo ME, Scherer PE. Adipose tissue-derived factors: impact on health and disease. Endocr Rev 2006;27:762-78.

10. Gandhi R, Takahashi M, Smith H, Rizek R, Mahomed NN. The synovial fluid adiponectin-leptin ratio predicts pain with knee osteoarthritis. Clin Rheumatol 2010;29:1223-8.

11. Maeda T, Kiguchi N, Kobayashi Y, Ikuta T, Ozaki M, Kishioka S. Leptin derived from adipocytes in injured peripheral nerves facilitates development of neuropathic pain via macrophage stimulation. Proc Natl Acad Sci U S A 2009;106:13076-81.

12. Massengale M, Lu B, Pan JJ, Katz JN, Solomon DH. Adipokine hormones and hand osteoarthritis: radiographic severity and pain. PLoS One 2012;7:e47860.

13. Franklin J, Ingvarsson T, Englund M, Lohmander LS. Sex differences in the association between body mass index and total hip or knee joint replacement resulting from osteoarthritis. Ann Rheum Dis 2009;68:536-40.

14. Karvonen-Gutierrez CA, Sowers MR, Heeringa SG. Sex dimorphism in the association of cardiometabolic characteristics and osteophytes-defined radiographic knee osteoarthritis among obese and non-obese adults: NHANES III. Osteoarthritis Cartilage 2012;20:614-21.

15. Yusuf E, Kloppenburg M. Epidemiological studies on adipokines and osteoarthritis. Int J Clin Rheumatol 2013;8:327.
16. Vuolteenaho K, Koskinen A, Kukkonen M, Nieminen R, Paivarinta $\mathrm{U}$, Moilanen T, et al. Leptin enhances synthesis of proinflammatory mediators in human osteoarthritic cartilage - mediator role of NO in leptin-induced PGE2, IL-6, and IL-8 production. Mediators Inflamm 2009;2009:345838.

17. Koskinen A, Vuolteenaho K, Nieminen R, Moilanen T, Moilanen E. Leptin enhances MMP-1, MMP-3 and MMP-13 production in human osteoarthritic cartilage and correlates with MMP-1 and MMP-3 in synovial fluid from OA patients. Clin Exp Rheumatol 2011;29:57-64.

18. Karvonen-Gutierrez CA, Harlow SD, Mancuso P, Jacobson J, Mendes de Leon CF, Nan B. Association of leptin levels with radiographic knee osteoarthritis among a cohort of midlife women. Arthritis Care Res 2013;65:936-44.

19. Staikos C, Ververidis A, Drosos G, Manolopoulos VG, Verettas DA, Tavridou A. The association of adipokine levels in plasma and synovial fluid with the severity of knee osteoarthritis. Rheumatology 2013;52:1077-83.

20. Ding C, Parameswaran V, Cicuttini F, Burgess J, Zhai G, Quinn S, et al. Association between leptin, body composition, sex and knee cartilage morphology in older adults: the Tasmanian older adult cohort (TASOAC) study. Ann Rheum Dis 2008;67:1256-61.

21. Stannus OP, Jones G, Quinn SJ, Cicuttini FM, Dore D, Ding C. The association between leptin, interleukin-6, and hip radiographic osteoarthritis in older people: a cross-sectional study. Arthritis Res Ther 2010;12:R95.

22. Laurberg TB, Frystyk J, Ellingsen T, Hansen IT, Jorgensen A, Tarp $\mathrm{U}$, et al. Plasma adiponectin in patients with active, early, and chronic rheumatoid arthritis who are steroid- and disease-modifying antirheumatic drug-naive compared with patients with osteoarthritis and controls. J Rheumatol 2009;36:1885-91

23. Filkova M, Liskova M, Hulejova H, Haluzik M, Gatterova J, Pavelkova A, et al. Increased serum adiponectin levels in female patients with erosive compared with non-erosive osteoarthritis. Ann Rheum Dis 2009;68:295-6.

24. de Boer TN, van Spil WE, Huisman AM, Polak AA, Bijlsma JW, Lafeber FP, et al. Serum adipokines in osteoarthritis; comparison with controls and relationship with local parameters of synovial inflammation and cartilage damage. Osteoarthritis Cartilage 2012;20:846-53.

25. Choe JY, Bae J, Jung HY, Park SH, Lee HJ, Kim SK. Serum resistin level is associated with radiographic changes in hand osteoarthritis: cross-sectional study. Joint Bone Spine 2012; 79:160-5.

26. Koskinen A, Vuolteenaho K, Nieminen R, Moilanen T, Moilanen E. Association of adipokines adipsin, resistin and leptin with interleukin 6 and metalloproteinases 1 and 3 in synovial fluid from osteoarthritis patients (abstract). Scand J Rheumatol 2012;41:327.

27. Fernández-Puente P, Mateos J, Fernández-Costa C, Calamia V, Ruiz-Romero C, Fernández-Lopez C, et al. Proteomic identification of a panel of novel osteoarthritis biomarkers in serum and cartilage (abstract). Arthritis Rheum 2011;63 Suppl 10:S1000. 